\title{
Coherent coupling of a trapped electron to a distant superconducting microwave cavity
}

Cite as: Appl. Phys. Lett. 117, 154001 (2020); https://doi.org/10.1063/5.0023002

Submitted: 25 July 2020 . Accepted: 30 September 2020 . Published Online: 13 October 2020

(iD) April Cridland Mathad, (D) John H. Lacy, (iD) Jonathan Pinder, (D) Alberto Uribe, (iD) Ryan Willetts, (i) Raquel Alvarez, and (iD) José Verdú

\section{COLLECTIONS}

Paper published as part of the special topic on Hybrid Quantum Devices HQD2021
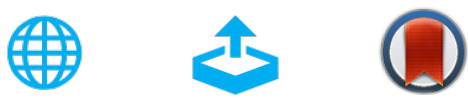

View Online

\section{ARTICLES YOU MAY BE INTERESTED IN}

Planar, strong magnetic field source for a chip ion trap

Review of Scientific Instruments 91, 103201 (2020); https://doi.org/10.1063/5.0024735

Quantum neuromorphic computing

Applied Physics Letters 117, 150501 (2020); https://doi.org/10.1063/5.0020014

Photon-mediated entanglement scheme between a ZnO semiconductor defect and a trapped Yb ion

Applied Physics Letters 117, 154002 (2020); https://doi.org/10.1063/5.0019892

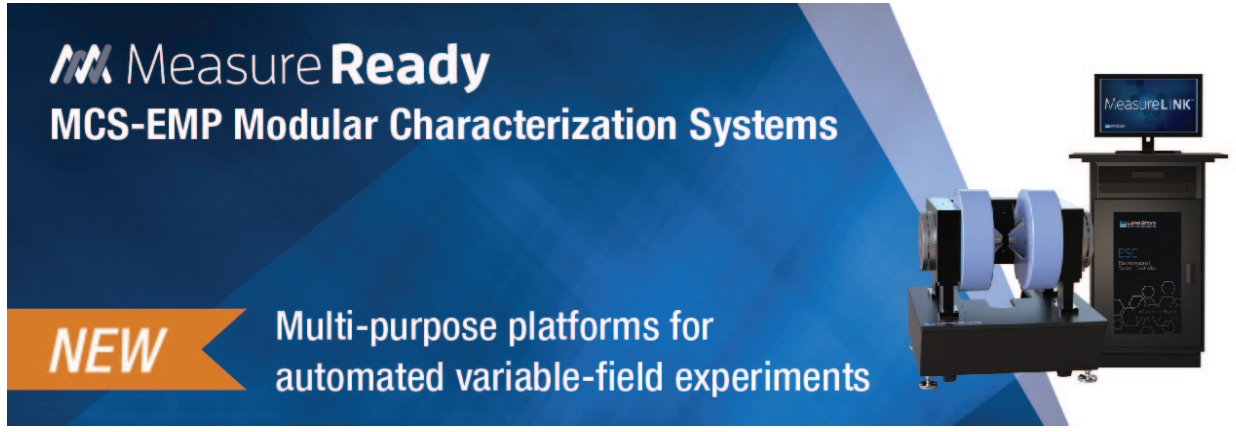

\section{Lake Shore}

\section{MCS-EMP Modular Characterization Systems}




\title{
Coherent coupling of a trapped electron to a distant superconducting microwave cavity
}

\author{
Cite as: Appl. Phys. Lett. 117, 154001 (2020); doi: 10.1063/5.0023002 \\ Submitted: 25 July 2020 - Accepted: 30 September 2020 . \\ Published Online: 13 October 2020
}

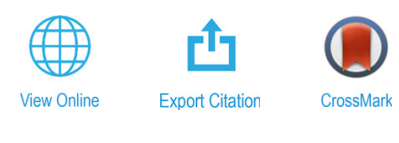

April Cridland Mathad, ' (D) John H. Lacy, ${ }^{2}$ (D) Jonathan Pinder, ${ }^{3}$ (D) Alberto Uribe, ${ }^{3}$ (D) Ryan Willetts, ${ }^{3}$ Raquel Alvarez, ${ }^{3}$ (D) and José Verdú ${ }^{3, a)}$ (D)

\author{
AFFILIATIONS \\ ${ }^{7}$ Swansea University, Singleton Campus, Swansea SA2 8PP, United Kingdom \\ ${ }^{2}$ Williams College, Williamstown, Massachusetts 01267, USA \\ ${ }^{3}$ Department of Physics and Astronomy, University of Sussex, Falmer BN1 9QH, United Kingdom
}

Note: This paper is part of the Special Issue on Hybrid Quantum Devices.

a) Author to whom correspondence should be addressed: jlv20@sussex.ac.uk

\begin{abstract}
We theoretically investigate the coupling of a single electron in a planar Penning trap with a remote superconducting microwave (MW) cavity. Coupling frequencies around $\Omega=2 \pi \cdot 1 \mathrm{MHz}$ can be reached with resonators with a loaded quality factor of $Q=10^{5}$, allowing for the strong coupling regime. The electron and the cavity form a system of two coupled quantum harmonic oscillators. This is a hybrid and linear microwave quantum network. We show that the coherent interaction can be sustained over distances of a few $\mathrm{mm}$ up to several $\mathrm{cm}$. Similar to classical linear MW circuits, the coherent quantum exchange of photons is ruled by the impedances of the electron and the cavity. As one concrete application, we discuss the entanglement of the cyclotron motions of two electrons located in two separate traps.
\end{abstract}

Published under license by AIP Publishing. https://doi.org/10.1063/5.0023002

Cryogenic Penning traps allow for a very accurate control of the dynamics of a trapped electron, at the fundamental level of quantum jumps between the Fock states of the harmonic trapping potential. ${ }^{1}$ The particles can be captured for very long periods (months); the continuous Stern-Gerlach effect permits the detection and manipulation of the electron's spin, ${ }^{2}$ and the Purcell effect enhances the coherence time of its motional quantum state. ${ }^{3}$ The latter can be monitored nondestructively, thereby effectively performing a quantum nondemolition (QND) measurement of microwave (MW) quanta. ${ }^{1}$ Hence, cryogenic Penning traps are excellent quantum laboratories and trapped electrons are solid candidates for quantum technology. ${ }^{4-6} \mathrm{We}$ are developing a trapped electron for quantum metrology applications, specifically as a transducer of quantum microwave (MW) radiation. ${ }^{7}$ For this, we use the "geonium chip" planar Penning trap. ${ }^{8-10}$ The electron can operate both as a detector and also as an emitter of MW photons. Being a quantum harmonic oscillator, in principle, its state can be mapped one-to-one onto the quantum state of a single mode MW radiation field. ${ }^{11}$ This makes the electron a linear and reversible quantum microwave transducer, unlike single MW photon counters based upon three-level systems ${ }^{12,13}$ and similar technologies. In those cases, only MW fields consisting of one (and only one) photon can be observed, and these are irreversibly lost after the detection. In contrast, the trapped electron might "witness" a more complex quantum microwave field and further reuse or redistribute it to other devices within a MW quantum network. In this Letter, we theoretically investigate the basic scheme for such reversible and QND measurements of MW radiation. The quantum object to be "measured" is assumed to be a distant superconducting microwave cavity, coupled to the electron through a transmission line of some finite length.

Figure 1(a) shows a geonium chip with its five basic trapping electrodes. These result from the projection of a five pole cylindric Penning trap onto a flat surface. ${ }^{8}$ The buried wires shown in Fig. 1(b) provide the required DC trapping voltages and also coupling for radio frequency $(\mathrm{RF})$ signals. They are connected through vias to the trap's electrodes. A static magnetic field, $\vec{B}=B_{0} \hat{u}_{z}$, forces the electron to follow a closed (cyclotron) orbit around its axis. The magnetic field source is shown in Fig. 1(c). It is made of NbTi superconducting wire and spans about $\sim 10 \times 10 \mathrm{~cm}^{2}$. Its construction and calibration have been described in Refs. 14 and 15. It is placed underneath the geonium chip and is constantly powered with DC supplies. An example of a measured field is shown in Fig. 1(d). Homogeneous magnetic fields up to $0.5 \mathrm{~T}(=5000 \mathrm{G})$ at the electron's trapping position can be reached with this source. A new magnetic source is under development, ${ }^{15}$ which operates in persistent mode and is magnetized with an especially devised flux pumping technique. ${ }^{16}$ The electrons can be captured at some height $y_{0}$ above the central conducting strip. The value of $y_{0}$ is 

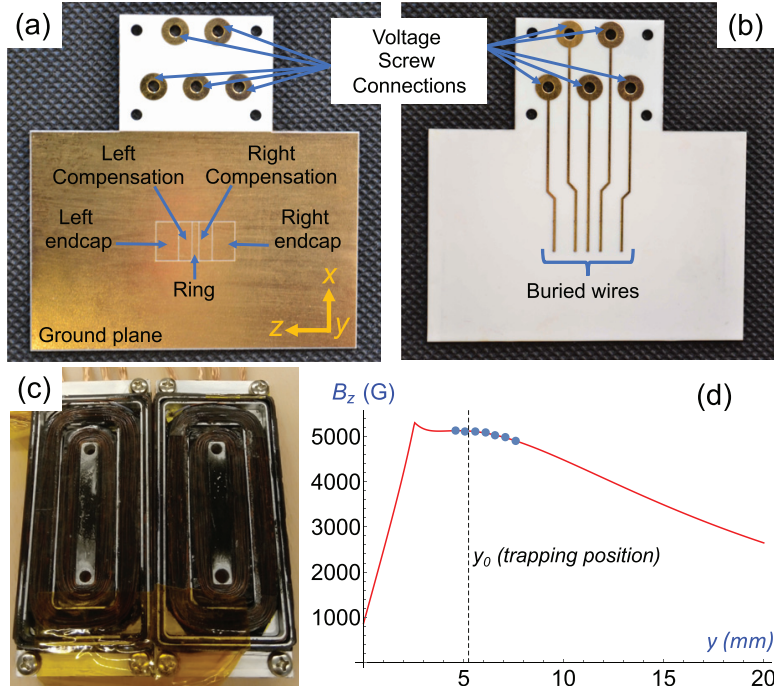

FIG. 1. (a) Top view of the geonium chip with the trap's electrodes. (b) Chip's rear side showing the buried wires. (c) Magnetic field source made of NbTi wire. (d) Example of the measured magnetic field (dots) and fitted Biot-Savart functions (continuous curve).

determined by the applied DC voltages. ${ }^{8}$ The particle's motion consists of three independent oscillations: the mentioned cyclotron motion, with frequency $\omega_{p}$, and the axial and magnetron motions. The latter two will not be further considered in this Letter. At $B_{0}=0.5 \mathrm{~T}$, we have $\omega_{p} / 2 \pi=13.99 \mathrm{GHz}$.

Superconducting microwave resonators can be fabricated in different shapes, for instance, as 3D cavities made of aluminium ${ }^{17}$ or copper with a tin coating, ${ }^{18}$ reaching internal quality factors as high as $Q \sim 10^{9}$, and also as flat, chip devices, such as coplanar-waveguide (CPW) cavities, achieving quality factors $Q \in\left[10^{5}, 10^{6}\right] .^{19,20}$ While our electron might be coupled to either kind of resonator, for simplicity, we concentrate on the CPW cavities; our analysis will apply also for other types of MW resonators. Their high $Q$ allows for transferring quantum information between different components in a microwave quantum circuit, such as in circuit-quantum electrodynamics (cQED). ${ }^{21}$ Due to the long coherence time of their spin state, electrons in different forms, such as trapped on the surface of liquid helium, within molecular ions, or as spin ensembles in solid state systems, have been proposed and are being tested as quantum memories for CQED. ${ }^{22-26} \mathrm{~A}$ review ${ }^{27}$ of other atomic systems proposed as potential quantum memories for $\mathrm{CQED}$ has been published. A scheme for interfacing electrons in a Paul trap with superconducting qubits has also been discussed. ${ }^{28}$ Our trapped electron can be coupled to a microwave quantum circuit via the interaction of the photons with its cyclotron motion or its spin. The latter is very weakly coupled, and hence, we focus on the former.

The electrodes of the geonium chip define the central conducting strip of a CPW transmission line, ${ }^{8}$ where MW photons can exist in either one of the two possible propagation modes, ${ }^{29}$ the odd or the even mode, of a CPW line. Thus, our chip might be designed and operated also as a planar microwave cavity. However, the quality factor of a superconducting CPW resonator is reduced by three to four orders of magnitude in the presence of a magnetic field. ${ }^{19}$ This makes it inadequate for storing MW photons. Furthermore, we want to investigate the QND measurement of the quantum state of a microwave system at an arbitrary distant location. Thus, we assume that our chip is coupled to a remote CPW superconducting cavity, as sketched in Fig. 2(a). The trap and the cavity are connected through a superconducting transmission line of some finite length $L_{\mathrm{TL}}$. The geonium chip confines the magnetic field into the trapping region, which decays rapidly away from it, as shown in Fig. 1(d). Hence, $\vec{B}$ does not reduce the quality factor of the superconducting cavity. The interaction between the distant MW quantum device (the cavity) and the electron is mediated by photons. For simplicity, we assume that the photons when arrived at the geonium chip exist in one mode of the CPW shaped by the trap's electrodes, as mentioned above. However, in general, this radiation can be delivered to the trapped electron by other types of transmission lines coupled to the electrodes or to a small antenna fabricated in the chip, such as the antennas used in near field scanning microwave microscopes. ${ }^{30}$

The interaction of a trapped electron with a radio frequency (RF) resonator made of lumped elements was first investigated by Dehmelt and Walls. ${ }^{31}$ This interaction is commonly employed for the electronic detection of the trapped particles and can be described as the coupling of two equivalent electric circuits. ${ }^{31}$ In our system of Fig. 2(a), the RF resonator is substituted by the superconducting CPW microwave cavity. The latter is equivalent to a parallel tank circuit, with inductance $L$, capacitance $C$, and losses modeled by the resistance $R$. Moreover, the electron's cyclotron motion acts as a series tank circuit, with equivalent inductance $L_{e}$ and capacitance $C_{e}{ }^{32}$ The electron-cavity interaction corresponds to the coupling in parallel to their equivalent electric circuits, ${ }^{31}$ as represented in Fig. 2(b). Hence, the system's overall impedance is $\frac{1}{Z_{\mathrm{L}}(\omega)}=\frac{1}{Z_{\text {cavity }}(\omega)}+\frac{1}{Z_{e}(\omega)}$, where $Z_{\text {cavity }}(\omega)$ is the cavity's input impedance seen from the trap and $Z_{e}(\omega)=i \omega L_{e}+\frac{1}{i \omega C_{e}}$ the electron's cyclotron impedance. $Z_{\mathrm{L}}(\omega)$ delivers a resonance spectrum as plotted in Fig. 2(c). In the case of resonant coupling, two symmetric peaks appear around the cavity's resonance frequency, $\omega_{\text {cavity }}$. The energy exchange rate between the particle and the resonator is given by the width $\Omega$ of the dip between both maxima. In Penning trap experiments such as in Refs. $33-35$, typical values of the used RF resonators ${ }^{36}$ are $Q \leq 10^{4}$. Thus, in

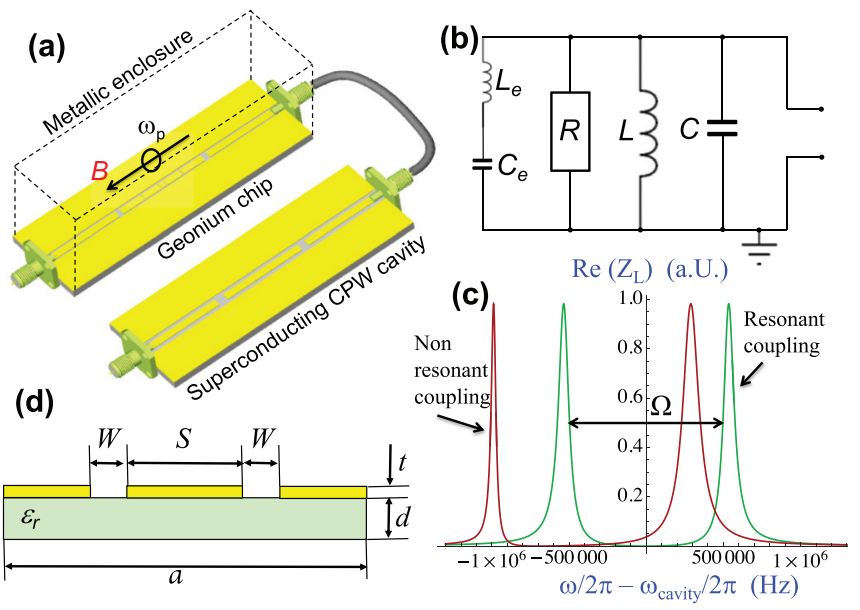

FIG. 2. (a) Sketch of the geonium chip connected to a remote superconducting CPW cavity. The open ends avoid photon leaks by reflecting the microwaves inside the system. (b) Equivalent electric circuit of the coupled electron and CPW cavity. (c) Impedance of the cavity + electron's cyclotron. This example assumes strong, coherent coupling. (d) Transverse dimensions of a CPW transmission line. 
those cases, once delivered to the resonator, the particle's energy is irreversibly dissipated almost instantly ${ }^{37}$ and $\tau=1 / \Omega$ is denoted as the "resistive cooling time constant."

In order to get the geonium chip down to $80 \mathrm{mK}$, we use a miniature adiabatic demagnetization refrigerator. ${ }^{38}$ This will also require MW attenuators $^{39,40}$ to avoid residual thermal photons entering into the system. With these, at $80 \mathrm{mK}$, the number of blackbody photons at 13.99 GHz is sufficiently low for both constituents of Fig. 2(a) to reach the ground state. ${ }^{1}$ This system becomes a quantum circuit and can be excited with a quantum of current, i.e., one single microwave photon. The photon then oscillates between both components at the coupling frequency $\Omega$. From the general expression of $\tau$, ${ }^{32}$ we have $\Omega(\delta)=\frac{\left|Z_{\text {cavity }}(\delta)\right|}{L_{e}}$, with $\delta=\omega_{\text {cavity }}-\omega_{p}$. The coupling $\Omega$ is maximum when the electron and the cavity are resonant $\omega_{\text {cavity }}=\omega_{p}$. In that case, the impedance of the cavity is $Z_{\text {cavity }}(0)=R=Q \omega_{\text {cavity }} L{ }^{41}$ When $\delta \neq$ $0,\left|Z_{\text {cavity }}(\delta)\right|$ decreases, the coupling strength is reduced, and the two maxima become asymmetric, as shown in Fig. 2(c). For the $\mathrm{e}^{-}$-cavity coupling to be coherent, $\Omega$ must be faster than the photon loss rate of the resonator, responsible for the irreversible energy dissipation causing the "resistive cooling." The cavity's photon loss rate is given by the width of its resonance: $\Gamma=\omega_{\text {cavity }} / Q$. Hence, the condition for coherent coupling is $\frac{\Omega}{\Gamma} \gg 1$. For the resonant case, $\delta=0$, we have

$$
\frac{\Omega}{\Gamma}=Q^{2} \frac{L}{L_{e}} \gg 1 .
$$

In Eq. (1), $Q$ is the quality factor of the loaded MW cavity, as seen by the electron, that is, including possible photon losses along the connecting transmission line. It must be observed that, as sketched in Fig. 2(a), the trap is enclosed within a rectangular metallic box. ${ }^{14}$ This also acts as a microwave resonator far detuned from $\omega_{p} \cdot{ }^{10}$ Hence, it prevents the electron from emitting cyclotron radiation into free space or into any other modes but those of the trap's CPW line, coupling to the remote MW cavity.

The ratio $\Omega / \Gamma$ of Eq. (1) can be calculated with the general expression ${ }^{7,32} L_{e}=\frac{m}{q^{2}} D_{\text {eff }}^{2}$. The symbols $q$ and $m$ represent the charge and mass of the electron, respectively. The "effective coupling distance," $D_{\text {eff }}$, is inversely proportional to the strength of the electric field at the position of the electron, $E_{\mathrm{CPW}}\left(y_{0}\right)$, of a $1 \mathrm{~V}$ microwave propagating along the CPW. In general, both quantities are related through ${ }^{32}$ $E_{\mathrm{CPW}}\left(y_{0}\right)=\frac{1 \mathrm{~V}}{D_{\text {eff }}}$. For the slow axial motion $\omega_{z}$, the corresponding RF electric field has been calculated using electrostatic techniques. ${ }^{32}$ That approach must be modified for the electron's cyclotron motion, which belongs to the MW domain. The electric field components $E_{\mathrm{CPW}}^{\text {odd }}$ and $E_{\mathrm{CPW}}^{\text {even }}$ have been computed analytically using special MW techniques. ${ }^{29}$ With those results, we obtain the cyclotron effective coupling distances, for each of the two possible CPW modes,

$$
\begin{aligned}
\left(D_{\text {eff }}^{\text {odd }}\right)^{-1}= & \frac{4}{\pi W} \sum_{n=1}^{\infty}\left\{\frac{\sin \left(n \pi \frac{W}{a}\right) \sin \left(n \pi \frac{W+S}{a}\right)}{\sqrt{n^{2}+\frac{a^{2}}{\lambda_{p}^{2}}-\frac{a^{2} \nu_{p}^{2}}{c^{2}}}}\right. \\
& \times \exp \left(-\frac{2 \pi y_{0}}{a} \sqrt{\left.n^{2}+\frac{a^{2}}{\lambda_{p}^{2}}-\frac{a^{2} \nu_{p}^{2}}{c^{2}}\right)}\right\},
\end{aligned}
$$

$$
\begin{aligned}
\left(D_{\text {eff }}^{\text {even }}\right)^{-1}= & \frac{4}{\pi W} \sum_{n=0}^{\infty}\left\{\frac{\sin \left(\frac{2 n+1}{2} \pi \frac{W}{a}\right) \cos \left(\frac{2 n+1}{2} \pi \frac{W+S}{a}\right)}{\frac{2 n+1}{2}}\right. \\
& \times \exp \left(-\frac{2 \pi y_{0}}{a} \sqrt{\left.\left.\left(\frac{2 n+1}{2}\right)^{2}+\frac{a^{2}}{\lambda_{p}^{2}}-\frac{a^{2} \nu_{p}^{2}}{c^{2}}\right)\right\} .}\right.
\end{aligned}
$$

In Eqs. (2) and (3) $c$ is the speed of light in vacuum. The transverse dimensions of the CPW are defined in Fig. 2(d). $S$ is the width of the central conducting strip, $W$ is the gap between the latter and the "ground planes," and $a$ is the total chip's width. In the geonium chip, the wavelength $\lambda_{p}$ of the radiation of cyclotron frequency $\left(\nu_{p}=\omega_{p} / 2 \pi\right)$ depends on the substrate's electric permittivity $\epsilon_{r}$, its thickness $d$, the thickness of the conducting layer $t$, and the dimensions $S, W .{ }^{42}$

In order to illustrate our system, we have calculated in detail one example. It is shown in Fig. 3. We have assumed a distant superconducting $\lambda / 4$ short circuited CPW microwave cavity, ${ }^{41}$ with dimensions: $S_{\text {cavity }}=10 \mu \mathrm{m}, W_{\text {cavity }}=4.8 \mu \mathrm{m}, t_{\text {cavity }}=200 \mathrm{~nm}$, and a sapphire substrate of $d_{\text {cavity }}=0.3 \mathrm{~mm}$. These values are motivated by some real resonators. ${ }^{19,20}$ Our cavity has the characteristic impedance of $Z_{0}=50 \Omega$, as obtained from previously compiled CPW design formulas. ${ }^{42}$ Further, we assume a loaded quality factor (as seen by the electron) of $Q=100000$. This corresponds to $\Gamma / 2 \pi=140 \mathrm{kHz}$, a resonance resistance of $R=6.37 \mathrm{M} \Omega$, and an inductance of $L=0.72$ $\mathrm{nH}$. Moreover, we have assumed a geonium chip of the same conducting layer and an alumina substrate of thickness $0.7 \mathrm{~mm}$, as shown in Fig. 1(a). With these, we compute $\Omega / \Gamma$ for 4 values of the width: $S_{\text {trap }}=0.5,0.25,0.15$, and $0.1 \mathrm{~mm}$, with gaps $W_{\text {trap }}=59,34,22$, and $15 \mu \mathrm{m}$, respectively. Each $W_{\text {trap }}$ is adjusted to ensure that the CPW formed in the geonium chip has a characteristic impedance of $Z_{0}=50 \Omega$.

The increasing values of $\Omega / \Gamma$ for smaller $S_{\text {trap }}$ observed in Figs. 3 (b) and 3(c) reflect the bigger strength, close to the chip's surface, of the microwave electric field $E_{\mathrm{CPW}}$ when the "mode volume" of the CPW line in the trap is reduced. At very low positions, $\Omega / \Gamma$ is higher for the odd than for the even mode; the latter actually disappears at $y_{0} \rightarrow 0$. This is due to the vanishing electric field component parallel to the conducting surface: $E_{\mathrm{CPW}}^{x} \rightarrow 0$ at $y_{0} \rightarrow 0$. The active mode, odd or even, depends on how the system is connected and grounded. ${ }^{42}$

\section{(a) Odd mode}

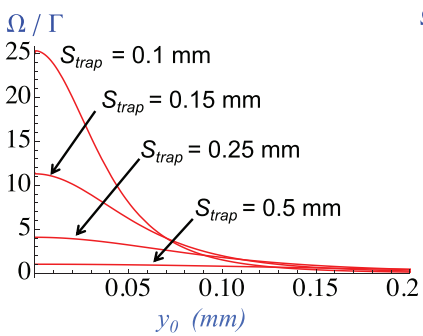

\section{(b) Even mode}

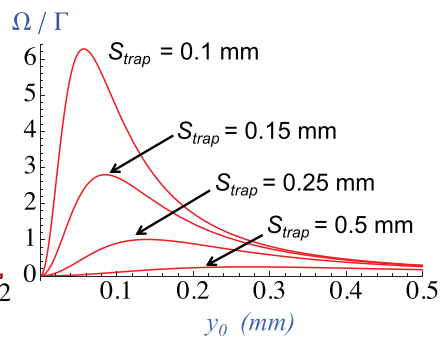

FIG. 3. Coupling frequency of one electron vs photon losses as a function of the trapping position, for various values of the CPW width in the trap chip. We assume $\delta=0$ and $Q=10^{5}$. Calculated with Eqs. (2) and (3), truncating the series at $n=60000$. (a) Odd mode. (b) Even mode. 
The electron can be positioned at $y_{0} \geq 50 \mu \mathrm{m}$, by carefully choosing the trapping voltages. ${ }^{8,10}$ At such low positions, the electrostatic trapping potential becomes anharmonic, ${ }^{8}$ and at $80 \mathrm{mK}$, the expected relative frequency shifts can be estimated to $\frac{\Delta \omega_{p}}{\omega_{p}} \simeq 6 \times 10^{-11}$. Such fluctuations of $\omega_{p}$ are negligibly small. Therefore, when placed at $y_{0} \geq 50 \mu \mathrm{m}$, the electron's cyclotron motion still acts as a quantum harmonic oscillator. As shown in Fig. 3 , at such low $y_{0}$, the cavity and the cyclotron oscillator interact coherently. After exchanging quantum information, $y_{0}$ can be increased (typically to $0.5-1 \mathrm{~mm}$ ) where the trapping potential becomes harmonic. There, $\omega_{p}$ can be measured accurately ${ }^{8,10}$ and the cyclotron quantum state can be read out and further manipulated.

The electron and the cavity are two coupled quantum harmonic oscillators. This is a linear system, where the coupling strength $\Omega$ is invariant under a number of photons. The coherent dynamics of two coupled quantum harmonic oscillators has been calculated. ${ }^{11}$ An equivalent system has been implemented with two ions held in two Paul traps at a distance of $40 \mu \mathrm{m} .{ }^{43}$ In our case, the maximum cavity-electron separation permitting coherent coupling is bound by the achievable loaded quality factor $Q$. The dependence $Q=Q\left(L_{\mathrm{TL}}\right)$ can be obtained from the transmission-line impedance equation. ${ }^{41}$ Thus, at the trap, the cavity's unloaded input impedance $Z_{\text {cavity }}^{0}(\omega)$ is transformed into

$$
Z_{\text {cavity }}\left(\omega \mid L_{\mathrm{TL}}\right)=Z_{0} \frac{Z_{\text {cavity }}^{0}(\omega) \operatorname{coth}\left(\gamma L_{\mathrm{TL}}\right)+Z_{0}}{Z_{0} \operatorname{coth}\left(\gamma L_{\mathrm{TL}}\right)+Z_{\text {cavity }}^{0}(\omega)} .
$$

The imaginary part of the propagation constant, $\gamma=\alpha+i \beta$, shifts the cavity's resonance frequency. We, therefore, assume that the cavity, as seen from the trap, has been designed to be resonant with $\omega_{p}$. The real part $\alpha$ accounts for the photon losses. The dissipation caused by the walls of the connecting transmission line is linked to the superconductor surface resistivity: $R_{S} \leq \frac{5}{4 \pi^{2}} \cdot 10^{-28} \omega^{2} \mathrm{Ohm}$ (with $\omega$ given in $\mathrm{rad} /$ s). ${ }^{44}$ At $13.99 \mathrm{GHz}$, it is negligible when compared to the dielectric losses. The latter, in the case of a superconducting coaxial line operating at $\omega_{p}$, gives rise to the attenuation constant $\alpha=\omega_{p}$ $\sqrt{\mu_{0} \cdot \epsilon_{\mathrm{TL}}} \tan \delta$, ${ }^{41}$ with $\mu_{0}$ being the magnetic permeability, $\epsilon_{\mathrm{TL}}$ the electric permittivity, and $\tan \delta$ the loss tangent. With that $\alpha$ and with the same cavity as in Fig. 3, assuming ${ }^{20} Q_{\text {unloaded }}=10^{6}$, we compute $Q=Q\left(L_{\mathrm{TL}}\right)$ from the spectra $Z_{\text {cavity }}\left(\omega \mid L_{\mathrm{TL}}\right)$. The results are given in Fig. 4(a).
We have assumed quartz with $\epsilon_{\mathrm{TL}}=4.64$ (relative) and $\tan \delta=3 \times 10^{-6}$; YAG with $\epsilon_{\mathrm{TL}}=10.40$ and $\tan \delta=1 \times 10^{-7}$, and sapphire with $\epsilon_{\mathrm{TL}}=9.4$ and $\tan \delta=4 \times 10^{-8}$, (all values are measured at $\sim 10 \mathrm{~K}){ }^{45}$ From the figure, we observe that, for quartz, the loaded quality factor drops below $10^{5}$ at a distance of $L_{\mathrm{TL}} \simeq 0.8 \mathrm{~cm}$, while both YAG and sapphire sustain $Q>10^{5}$ at separations above $10 \mathrm{~cm}$. Within that range of distances, coherent $\mathrm{e}^{-}$-cavity coupling might be achieved, as shown in Fig. 3. It must be observed that in this discussion, we have assumed one or small numbers of MW photons. When the quantum state of the exchanged MW signal consists of a large number of photons, then the coherence or the "quantum nature" of the state will, in general, degrade more rapidly than for low photon numbers. ${ }^{46}$ A larger number of photons will make the exchanged MW signal increasingly nonmonochromatic, and in that case, the dispersion of the coupling transmission line will need to be considered.

Through the coherent interaction of the trapped electron with a remote superconducting resonator, the former can be further coupled to other components in a microwave quantum network. The electron's cyclotron oscillator enables the linear mapping of the quantum state of any multi photon microwave field onto an atomic degree of freedom. This is possible while keeping the photons stored within the cyclotron quantum state for periods of the order of a minute. ${ }^{1}$ The linearity of the electron also makes the computation of the coupling with any other quantum devices in a MW network straightforward: the coupling strength $\Omega$ is simply given by the transformed impedance [through Eq. (4)] of that device as seen by the electron. Furthermore, in contrast to systems where the atomic species are attached to the CPW microwave cavity, ${ }^{22-26}$ our electron quantum MW transducer and the cavity are two fully independent devices. This makes increasingly complex network topologies possible, enabling many applications beyond quantum MW memories. A fundamental one is the entanglement of two electrons stored in different traps. This can be achieved with a configuration as sketched in Fig. 4(b). There, two traps are connected to two cavities of the same resonance frequencies and quality factors. Cyclotron-cyclotron entanglement occurs through a similar mechanism as demonstrated with two laser-cooled ions in distant Paul traps. ${ }^{47}$ For that, cyclotron oscillators must be prepared in the first excited state, $n_{p}=1$, with both particles at a high $y_{0}$, i.e., initially invisible to the cavities, due to the vanishing coupling (Fig. 3). Such state initialization can be achieved by pumping photons from a
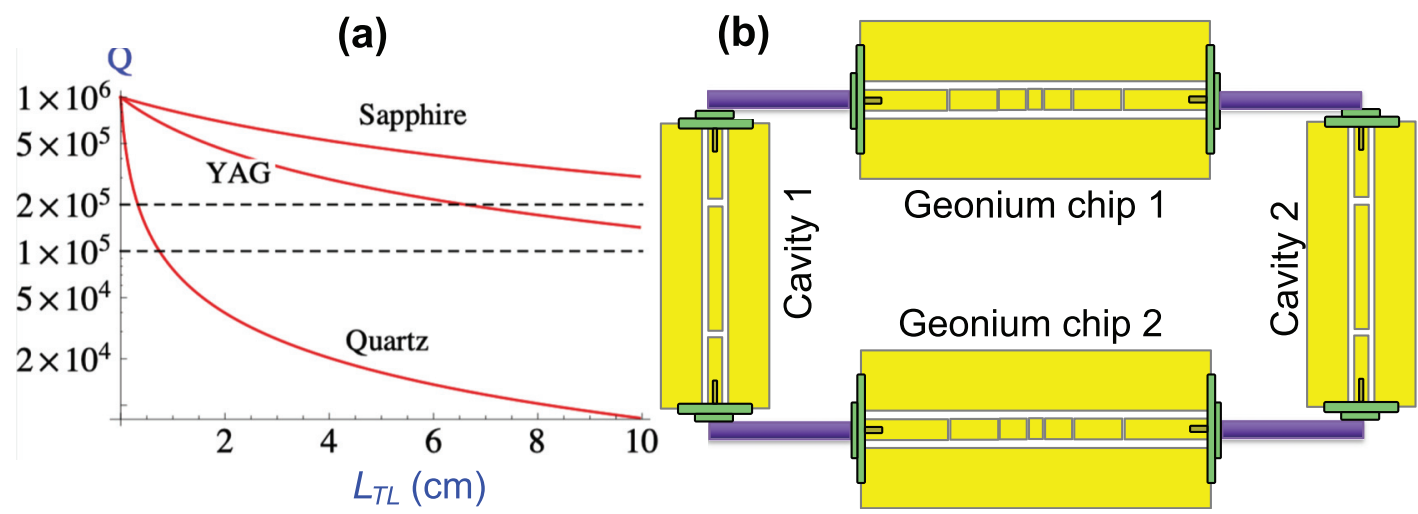

FIG. 4. (a) Loaded Q vs trap-cavity distance. Calculated for three different dielectric materials in the coax line. (b) Two geonium chips connected to two different distant identical cavities. 
very weak source connected to the traps, while monitoring the quantum number $n_{p}$ nondestructively. ${ }^{1}$ Thereafter, the electrons are simultaneously dropped for a short period of time to a very low position, around $50 \mu \mathrm{m}$, hence switching on briefly the strong coupling with the MW cavities. Hence, in our system, the unavailable fast spontaneous emission (essential in the Paul trap experiment ${ }^{47}$ ) is substituted by the rapidly switchable strong coupling to the remote resonators. This forces both cyclotrons to deliver the microwave photons simultaneously (in principle with equal probability) to any of the cavities. The irreversible detection of the emitted photons, either in cavity 1 or 2 (for instance as in Ref. 48), erases the "which way" information, thereby entangling the particles. The entanglement of electrons stored in one trap has been proposed for quantum metrology applications, such as the enhancement of the g-factor measurement. ${ }^{49}$ The trapped electron quantum microwave transducer can also be used in other practical applications, for instance, as an ultra-sensitive MW detector for near-field scanning microwave microscopes. ${ }^{50}$

This work was supported by EPSRC, through Grant Nos. EP/ N003675/1 and EP/R008558/1.

\section{DATA AVAILABILITY}

The data that support the findings of this study are available within this article.

\section{REFERENCES}

${ }^{1}$ S. Peil and G. Gabrielse, "Observing the quantum limit of an electron cyclotron: QND measurements of quantum jumps between Fock states," Phys. Rev. Lett. 83, 1287-1290 (1999).

${ }^{2}$ H. G. Dehmelt, "Continuous Stern-Gerlach effect: Principle and idealized apparatus,” Proc. Natl. Acad. Sci. U. S. A. 83, 2291-2294 (1986).

${ }^{3} \mathrm{G}$. Gabrielse and H. Dehmelt, "Observation of inhibited spontaneous emission,” Phys. Rev. Lett. 55, 67-70 (1985).

${ }^{4}$ G. Ciaramicoli, I. Marzoli, and P. Tombesi, "Scalable quantum processor with trapped electrons,” Phys. Rev. Lett. 91, 017901 (2003).

${ }^{5}$ G. Ciaramicoli, I. Marzoli, and P. Tombesi, "Quantum spin models with electrons in Penning traps,” Phys. Rev. A 78, 012338 (2008).

${ }^{6}$ G. Ciaramicoli, I. Marzoli, and P. Tombesi, "From a single- to a double-well Penning trap,” Phys. Rev. A 82, 044302 (2010).

${ }^{7}$ A. Cridland, J. Lacy, J. Pinder, and J. Verdú, "Single microwave photon detection with a trapped electron," Photonics 3, 59 (2016).

${ }^{8}$ J. Verdú, "Theory of the coplanar waveguide Penning trap," New J. Phys. 13, 113029 (2011).

9. Verdú, "Ion trap," patent WO 2013/041615 A2 (March 28, 2013).

${ }^{10} \mathrm{~J}$. Pinder and J. Verdú, "A planar Penning trap with tunable dimensionality of the trapping potential,” Int. J. Mass Spectrom. 356, 49 (2013).

${ }^{11}$ L. E. Estes, T. H. Keil, and L. M. Narducci, "Quantum-mechanical description of two coupled harmonic oscillators," Phys. Rev. 175, 286-299 (1968).

${ }^{12}$ G. Romero, J. J. García-Ripoll, and E. Solano, "Microwave photon detector in circuit QED,” Phys. Rev. Lett. 102, 173602 (2009).

${ }^{13}$ K. Inomata, Z. Lin, K. Koshino, W. D. Oliver, J.-S. Tsai, T. Yamamoto, and Y. Nakamura, "Single microwave-photon detector using an artificial [lambda]type three-level system," Nat. Commun. 7, 12303 (2016).

${ }^{14} \mathrm{~J}$. Pinder, "The geonium chip: Engineering a scalable planar Penning trap," Ph.D. thesis (University of Sussex, 2017).

${ }^{15} \mathrm{~J}$. H. Lacy, "Development of a planar magnetic field source for the geonium chip Penning trap,” Ph.D. thesis (University of Sussex, 2019).

${ }^{16}$ J. H. Lacy, A. Cridland, J. Pinder, A. Uribe, R. Willetts, and J. Verdú, "Superconducting flux pump for a planar magnetic field source," IEEE Trans. Appl. Supercond. 30, 1-12 (2020).

${ }^{17}$ M. Reagor, H. Paik, G. Catelani, L. Sun, C. Axline, E. Holland, I. Pop, N. Masluk, T. Brecht, L. Frunzio, M. Devoret, L. Glazman, and R. Schoelkopf,
"Reaching $10 \mathrm{~ms}$ single photon lifetimes for superconducting aluminum cavities," Appl. Phys. Lett. 102, 192604 (2013).

${ }^{18}$ D. Bogorin, M. Ware, D. McClure, S. Sorokanich, and B. Plourde, "Reducing surface loss in 3D microwave copper cavities for superconducting transmon qubits," in IEEE 14th International Superconductive Electronics Conference, ISEC (2013), p. 6604283.

${ }^{19}$ L. Frunzio, A. Wallraff, D. Schuster, J. Majer, and R. Schoelkopf, "Fabrication and characterization of superconducting circuit QED devices for quantum computation," IEEE Trans. Appl. Supercond. 15, 860-863 (2005).

${ }^{20}$ A. Megrant, C. Neill, R. Barends, B. Chiaro, Y. Chen, L. Feigl, J. Kelly, E. Lucero, M. Mariantoni, P. J. J. O’Malley, D. Sank, A. Vainsencher, J. Wenner, T. C. White, Y. Yin, J. Zhao, C. J. Palmström, J. M. Martinis, and A. N. Cleland, "Planar superconducting resonators with internal quality factors above one million," Appl. Phys. Lett. 100, 113510 (2012).

${ }^{21}$ A. Blais, R.-S. Huang, A. Wallraff, S. M. Girvin, and R. J. Schoelkopf, "Cavity quantum electrodynamics for superconducting electrical circuits: An architecture for quantum computation," Phys. Rev. A 69, 062320 (2004).

${ }^{22}$ D. I. Schuster, A. P. Sears, E. Ginossar, L. DiCarlo, L. Frunzio, J. J. L. Morton, H. Wu, G. A. D. Briggs, B. B. Buckley, D. D. Awschalom, and R. J. Schoelkopf, "High-cooperativity coupling of electron-spin ensembles to superconducting cavities," Phys. Rev. Lett. 105, 140501 (2010).

${ }^{23}$ D. I. Schuster, A. Fragner, M. I. Dykman, S. A. Lyon, and R. J. Schoelkopf, "Proposal for manipulating and detecting spin and orbital states of trapped electrons on helium using cavity quantum electrodynamics," Phys. Rev. Lett. 105, 040503 (2010)

${ }^{24}$ D. I. Schuster, L. S. Bishop, I. L. Chuang, D. DeMille, and R. J. Schoelkopf, "Cavity QED in a molecular ion trap," Phys. Rev. A 83, 012311 (2011).

${ }^{25} \mathrm{H}$. Wu, R. E. George, J. H. Wesenberg, K. Mølmer, D. I. Schuster, R. J. Schoelkopf, K. M. Itoh, A. Ardavan, J. J. L. Morton, and G. A. D. Briggs, "Storage of multiple coherent microwave excitations in an electron spin ensemble," Phys. Rev. Lett. 105, 140503 (2010).

${ }^{26}$ Y. Kubo, C. Grezes, A. Dewes, T. Umeda, J. Isoya, H. Sumiya, N. Morishita, H. Abe, S. Onoda, T. Ohshima, V. Jacques, A. Dréau, J.-F. Roch, I. Diniz, A. Auffeves, D. Vion, D. Esteve, and P. Bertet, "Hybrid quantum circuit with a superconducting qubit coupled to a spin ensemble," Phys. Rev. Lett. 107, 220501 (2011).

${ }^{27}$ Z.-L. Xiang, S. Ashhab, J. Q. You, and F. Nori, "Hybrid quantum circuits: Superconducting circuits interacting with other quantum systems," Rev. Mod. Phys. 85, 623-653 (2013)

${ }^{28}$ N. Daniilidis, D. J. Gorman, L. Tian, and H. Häffner, "Quantum information processing with trapped electrons and superconducting electronics," New J. Phys. 15, 073017 (2013).

${ }^{29}$ R. N. Simons and R. Arora, "Coupled slot line field components," IEEE Trans. Microwave Theory Tech. 30, 1094 (1982).

${ }^{30}$ S. Anlage, V. Talanov, and A. Schwartz, Principles of Near-Field Microwave Microscopy (Springer-Verlag, New York, 2007), Vol. 1, pp. 215-253.

${ }^{31}$ H. G. Dehmelt and F. L. Walls, "Bolometric technique for the RF spectroscopy of stored ions,” Phys. Rev. Lett. 21, 127-131 (1968).

${ }^{32} \mathrm{~A}$. Al-Rjoub and J. Verdú, "Electronic detection of a single particle in a coplanar-waveguide Penning trap,” Appl. Phys. B 107, 955 (2012).

${ }^{33}$ D. Hanneke, S. Fogwell, and G. Gabrielse, "New measurement of the electron magnetic moment and the fine structure constant," Phys. Rev. Lett. 100, 120801 (2008).

${ }^{34}$ S. Sturm, A. Wagner, B. Schabinger, J. Zatorski, Z. Harman, W. Quint, G. Werth, C. H. Keitel, and K. Blaum, "g factor of hydrogenlike ${ }^{28} \mathrm{Si}^{13+}$," Phys. Rev. Lett. 107, 023002 (2011).

${ }^{35}$ S. Ulmer, C. C. Rodegheri, K. Blaum, H. Kracke, A. Mooser, W. Quint, and J. Walz, "Observation of spin flips with a single trapped proton," Phys. Rev. Lett. 106, 253001 (2011).

${ }^{36}$ S. Ulmer, H. Kracke, K. Blaum, S. Kreim, A. Mooser, W. Quint, C. C. Rodegheri, and J. Walz, "The quality factor of a superconducting RF resonator in a magnetic field," Rev. Sci. Instrum. 80, 123302 (2009).

${ }^{37}$ W. M. Itano, J. C. Bergquist, J. J. Bollinger, and D. J. Wineland, “Cooling methods in ion traps," Phys. Scr. T59, 106-120 (1995).

${ }^{38} \mathrm{~J}$. Bartlett, G. Hardy, and I. Hepburn, "Performance of a fast response miniature adiabatic demagnetisation refrigerator using a single crystal tungsten magnetoresistive heat switch," Cryogenics 72, 111-121 (2015). 
${ }^{39}$ J.-H. Yeh, J. Lefebvre, S. Premaratne, F. Wellstood, and B. Palmer, "Microwave attenuators for use with quantum devices below $100 \mathrm{mK}$," J. Appl. Phys. 121, 224501 (2017).

${ }^{40}$ Z. Wang, S. Shankar, Z. Minev, P. Campagne-Ibarcq, A. Narla, and M. Devoret, "Cavity attenuators for superconducting qubits," Phys. Rev. Appl. 11, 014031 (2019).

${ }^{41}$ D. M. Pozar, Microwave Engineering (Wiley and Sons, 2004).

${ }^{42}$ B. C. Wadell, Transmission Line Design Handbook (Artech House, Norwood, MA, 1991).

${ }^{43}$ K. Brown, C. Ospelkaus, Y. Colombe, A. Wilson, D. Leibfried, and D. Wineland, "Coupled quantized mechanical oscillators," Nature 471, 196-199 (2011).

${ }^{44} \mathrm{~J}$. Winters and C. Rose, "High-Tc superconductor waveguides: Theory and applications," IEEE Trans. Microwave Theory Tech. 39, 617-623 (1991).

${ }^{45}$ J. Krupka, K. Derzakowski, M. Tobar, J. Hartnett, and R. G. Geyer, "Complex permittivity of some ultralow loss dielectric crystals at cryogenic temperatures," Meas. Sci. Technol. 10, 387 (1999).
${ }^{46}$ A. Chizhov, E. Schmidt, L. Knöll, and D.-G. Welsch, "Propagation of entangled light pulses through dispersing and absorbing channels," J. Opt. B 3, 77 (2001).

${ }^{47}$ D. L. Moehring, P. Maunz, S. Olmschenk, K. C. Younge, D. N. Matsukevich, L. M. Duan, and C. Monroe, "Entanglement of single-atom quantum bits at a distance," Nature 449, 68-71 (2007).

${ }^{48}$ B. R. Johnson, M. D. Reed, A. A. Houck, D. I. Schuster, L. S. Bishop, E. Ginossar, J. M. Gambetta, L. DiCarlo, L. Frunzio, S. M. Girvin, and R. J. Schoelkopf, "Quantum non-demolition detection of single microwave photons in a circuit," Nat. Phys. 6, 663-667 (2010).

${ }^{49}$ L. Lamata, D. Porras, J. I. Cirac, J. Goldman, and G. Gabrielse, "Towards electron-electron entanglement in Penning traps," Phys. Rev. A 81, 022301 (2010).

${ }^{50}$ S. Geaney, D. Cox, T. Hönigl-Decrinis, R. Shaikhaidarov, S. Kubatkin, T. Lindström, A. Danilov, and S. de Graaf, "Near-field scanning microwave microscopy in the single photon regime," Sci. Rep. 9, 12539 (2019). 